\title{
Ten commandments of hypospadias surgery
}

\begin{abstract}
Aims: It is to improve the overall $60 \%$ success rate in hypospadias surgery to a much higher level by few modifications done by me and others selected from vast options available in hypospadias surgery. I call these ten protocols which consistently gave results as the Ten Commandments.
\end{abstract}

Methods and material: First 7 years of my practice in Jawale Institute of Pediatric Surgery I did 257 cases of hypospadias surgery strictly as described by Dr. Warren Snodgrass for single stage repair and Dr. Byar's two staged urethroplasty for two staged repair. This group was kept as a control (group A) to be compared with the group of patients done with Ten Commandments (group B). Age range was 9 months to 13 years. Distal Hypospadias was found in 134(52\%), Mid penile in 77(30\%) and proximal Hypospadias in $46(18 \%)$ patients. Tubularized incised plate urethroplasty (TIP) by Dr. Warren Snodgrass and Byar's two stage urethroplasty were the only two basic methods applied. Only 13 patients (5\%) of total required two stage repairs. Subsequent 10 years, I operated 512 cases of hypospadias with the Ten Commandments. Tubularized incised plate urethroplasty (TIP) Warren Snodgrass repair and Byar's two stage urethroplasty were the only two basic methods applied. Distal Hypospadias was found in $230(45 \%)$, Mid penile in $180(35 \%)$ and proximal Hypospadias in $102(20 \%)$ patients. Only 23 patients $(4.5 \%)$ of total required two stage repair.

Results: The results of Tubularized incised plate urethroplasty (TIP) Warren Snodgrass repair are as follows: fistula rate of $10 \%$ and $21 \%$ respectively for distal and proximal hypospadias. Meatal Stenosis in 3\% cases. No patient had urethral stricture and glanular dehiscence. No patient had a residual chordee. Complication of two staged urethroplasty are fistula rate $24 \%$, meatal stenosis $3 \%$, no patients had residual chordee and complete disruption. The two staged urethroplasties done with buccal mucosa as an onlay graft are very bad with $50-100 \%$ failure, many centers have discontinued these repairs. Hence I did not perform any of these repairs in my series. The results of my series with 257 cases before the protocol(group A) were as follows: 33 patients (12.8\%) developed meatal stenosis for which only one urethral dilatation under GA was required. 78 patients(30.35\%) developed fistula and 18 of them closed spontaneously with conservative treatment and 60(23.34\%) required fistula repair. Total complication rate was $66.5 \%$.The results of my series with 512 cases done with Ten Commandments (group B) were as follows: 17 patients (3\%) developed meatal stenosis for which only one urethral dilatation under GA was required. Although 9 patients developed fistula 6 of them closed spontaneously with conservative treatment and only three required fistula repair $(0.6 \%)$. Total complication rate was $3.6 \%$.No patient developed a urethral stricture. Incidance of meatal stenosis, fistula formation, fistula closure operation and total complication rate of group A was compared to group B and all the complications were found to be far less frequent in group B compared to group A and the difference was found to be statistically significant. The results of group B were compared to the series of Warren Snodgrass and Aseem Shukla who are the international authorities on the subject and have written a chapter on Hypospadias in paediatric urology text book Kelalis and King. They have a fistula rate of $10 \%$ and $21 \%$ respectively for distal and proximal hypospadias which is 17 and 35 times higher than my series. Other international series on Hypospadias have more or less similar outcomes.

\section{Discussion}

\section{a. The Major Commandments are}

I. Taking continuous interlocking sutures and interrupting suture line by taking a knot every 5 stitches. This kind of suturing policy is reported for the first time in Hypospadias surgery literature. These sutures are water tight and if sutures breaks in between the whole suture line does not unwind.

II. Dartos flap raised from scrotum. It is robust, has excellent blood supply and gives support and protection to suture line avoiding fistula formation. Dartos is a vascular tissue which promotes healing.

III. Suprapubic diversion for three weeks. Healing takes about 3-4 weeks and
Volume 6 Issue 6 - 2017

Sagar A Jawale

Jawale Institute of Paediatric Surgery, India

Correspondence: Sagar A Jawale, Jawale Institute of Paediatric Surgery, India, Email drsagarjawale@gmail.com

Received: November 16, 2016 | Published: June 13, 2017

removing urethral stent in one week time is too early. Urine flows in force and pressure that can easily disrupt mucosal level of healing that occur at the end of one week. Collagen is laid down at the end of a third week which gives strength to the suture line. Just adding suprapubic diversion dropped the fistula rate by about $50 \%$.

IV. Rotating total prepucial skin instead of Byar's skin flaps. Dorsally cutting the prepucial hood can jeopardize the blood supply of either of skin flaps and it can get necrosed leading to failure of the operation. Another problem is that suture line of urethra crosses with the skin which is a violation of basic principles of surgery.

V. Tourniquet instead of adrenaline infiltration. Adrenaline can give false sense of no bleeding and post operatively big hematomas form that will disrupt the repair. If adrenaline accidently enters blood stream, it can lead to serious cardiac arrhythmias. Adrenaline can also lead to necrosis of the skin around areas of infiltration.

\section{b. Minor commandments are}

I. Dressing policy. The penis is covered with a sofra tulle and a gauze piece is applied over it. Compression dressing is given by Elastoplasts. It takes care of post operative bleeding and hematoma formation. Such a dressing also protects the repair from any possible injuries in the post operative period.

II. High protein diet in the form of eggs or protinex powder. Despite good Hypospadias repair I got some failures due to poor wound healing. I started high protein diet to the child before, during and after surgery in the form of eggs or protinex powder that made dramatic difference to healing and results.

III. Tab Oxybutynin given in twice a day schedule. Violent bladder contractions can lead to peri urethral gush of urine and a possible fistula formation.

IV. Using bipolar cautery to cauterize all major bleeders. If all major bleeders are not cauterized, post operatively a major hematoma can form that can destroy the repair. Hence only bipolar cautery should be used in Hypospadias surgery.

V. Ornidazole covers substantially dropped the infection rate

Conclusions: success rate of hypospadias surgery became $99.4 \%$. The complication rate in group B was far less than that of group A and it was found to be statistically very significant. Since the Author Warren Snodgrass and others did not follow any of the above Commandments in their series, there is a reason to follow them for a much better outcome. I believe that similar results are achievable by any pediatric surgeon by following the Ten Commandments.

Keywords: hypospadias, modified technique for hypospadias surgery, snodgrass repair 


\section{Introduction}

Hypospadias is a congenital disorder of the urethra where the urinary opening is not at the usual location on the tip of the penis. It is the second most common birth abnormality of the male reproductive system, affecting about one of every 250 males at birth. In roughly $90 \%$ of cases, the opening (meatus) is on or near the tip of the penis (glans), referred to as distal hypospadias, while the remainder have proximal hypospadias with a meatus near or within the scrotum. Shiny tissue seen extending from the meatus to the tip of the glans, which would have made the urinary channel, is referred to as the urethral plate. In most cases, the foreskin is also underdeveloped and does not wrap completely around the penis, leaving the underside of the glans penis uncovered. Also, a downward bending of the penis, commonly referred to as chordee, may occur. This is found in $10 \%$ of distal hypospadias and $50 \%$ of proximal hypospadias. The scrotum may be higher than usual to either side of the penis, called penoscrotal transposition, adding to the overall abnormal appearance. Hypospadias is thought to result from failure of the urinary channel to completely tubularize to the end of the penis; the cause is not known. Most often, it is the only abnormal finding, although in about $10 \%$ of cases, hypospadias may be part of a syndrome with multiple abnormalities.

The overall success rate in hypospadias surgery is not more than $60 \%$ in most of the hands. There are numerous methods described in medical literature for the Hypospadias surgery. Many of these methods are outdated and do not work. It is particularly confusing to junior surgeons as they don't know which methods work. As a novice in hypospadias surgery 17 years ago, I started doing the surgeries strictly as described by Dr. Warren Snodgross and Dr. Byar. My results were very poor with overall complication rate exceeding $50 \%$. In India majority of the population is not covered with medical insurance and the patients have to pay for the treatment from their pocket. It is said that necessity is the mother of all the inventions which applied to me in this situation. Patient dissatisfaction, high complication rate and survival in my practice were the reasons that I wanted to modify these operations for a much greater results. I wanted to improve the success rate of Hypospadias surgery to a much higher level for which I did a few modifications myself and others were selected from vast options available in medical literature on hypospadias surgery. The protocols and methods which work were kept and those which did not work were deleted from my protocol. I call these ten protocols which consistently gave results as the Ten Commandments. This trial and error method continued for 7 years till my protocol was ready. Next 10 years I practiced the Ten Commandments strictly on 512 cases and it was compared to the results of my previous 7 years results. God gave Moses Ten Commandments for a successful life. God also said to Moses that you just miss one commandment and I can't assure you Heaven. I had a similar experience here, I miss even one Commandment accidently today I have a failure, hence the name Ten Commandments.

\section{Materials and methods}

First 7 years of my practice in Jawale Institute of Pediatric Surgery I did 257 cases of hypospadias surgery strictly as described by Dr. Warren Snodgrass for single stage repair and Dr. Byar's two staged urethroplasty for two staged repair. This group was kept as a control (group A) to be compared with the group of patients done with Ten Commandments (group B). The study was done by following the national and international norms of medical ethics. The Institutional ethical clearance certificate was taken and attached along with this study. Age range was 9 months to 13 years. Glanular hypospadias cases were excluded. Distal Hypospadias was found in 134(52\%), mid penile in $77(30 \%)$ and proximal Hypospadias in $46(18 \%)$ patients. Tubularized incised plate urethroplasty (TIP) by Dr. Warren Snodgrass and Byar's two stage urethroplasty were the only two basic methods applied. Only 13 patients (5\%) of total required two stage repair.

Last 10 years, in Jawale Institute of Pediatric Surgery I operated 512 cases of hypospadias with the Ten Commandments (group B). Age range was 11 months to 15 years. Glanular hypospadias cases were excluded. Distal Hypospadias was found in 230(45\%), mid penile in $180(35 \%)$ and proximal Hypospadias in $102(20 \%)$ patients. Tubularized incised plate urethroplasty (TIP) by Dr. Warren Snodgrass and Byar's two stage urethroplasty were the only two basic methods applied. Only 23 patients (4.5\%) of total required two stage repairs.

\section{The Major Commandments are}

1) Taking continuous interlocking sutures and a knot every 5 th stitch

\section{2) Dartos flap}

3) Suprapubic diversion for three weeks.

4) Rotating total prepucial skin instead of Byar's skin flaps.

5) Tourniquet instead of adrenaline infiltration.

\section{The Minor Commandments are}

I. Dressing policy.

\section{High protein diet}

\section{Tab Oxybutynin}

\section{Using bipolar cautery to cauterize all major bleeders}

\section{Postoperative IV Ornidazole cover.}

Dr. Warren Snodgrass Tubularized incised plate urethroplasty (TIP) repair ${ }^{28,29}$ technique is as follows. The penis is degloved after a circumferential subcoronal incision is taken $2 \mathrm{~mm}$ proximally to the urethral native meatus. A U-shaped incision is taken along the lateral margins of the urethral plate. The glans wings are then created. A relaxing incision is the made in the midline of the urethral plate to allow tension free tubularization of the urethra. Tubularization itself is then performed with a $6 / 0$ running suture, subcuticular and non interlocking. A buttonholed dartos flap can be transposed from the dorsal side of the penis to the ventral side allowing coverage of the tubularized neo-urethra. Glans wings approximation starts the glanuloplasty at the corona. Suture of the skin edges and of the meatus finalizes the technique.

Dr. Warren Snodgrass in his Tubularized incised plate urethroplasty (TIP) repair ${ }^{1,2}$ uses continuous non interlocking sutures without taking knots in between. He raises Dartos flap from the prepucial hood and not from the scrotum. He wraps the skin over the penis by taking a dorsal midline cut called Byar's skin flaps. Suprapubic diversion is not done in his series. Other details such as use of cautery, tourniquet or adrenaline and postoperative antibiotic protocol and details of dressing policy are not mentioned by him in his paper: In conventional Byar's first stage operation, the procedure starts removing any fibrous tissue on the ventral aspect of the penis. Any poor quality distal urethra is removed too, whereas the urethral plate is initially spared. Glans wings are developed performing a deep incision in the midline and lifting glanular tissue off the apex of the penile corpora laterally. A 
chordee test is performed at the end of this step. If curvature persists, usually a dorsal placation is done. The penis is de gloved, dorsal neurovascular bundle isolated and tunica albuginea plicated in the midline after creating multiple small transverse incisions in tunica albuginea. As an alternative to dorsal plication, some authors prefer to perform a ventral grafting to correct the curvature. A third possibility is to achieve a ventral lengthening by performing multiple transversal incisions on the ventral aspect of the corpora and then to cover all with the free graft. In both, the latter the urethral plate is discarded at the beginning of the procedure. Next a proximal urethrostomy is performed. If the meatus is perineal, it is advanced at the level of the penoscrotal junction. The spatulated meatus is anchored to the corpus cavernosum and adjacent skin.

For Byar's conventional second stage urethroplasty ${ }^{3,7}$ the following technique is used. Two parallel incisions are taken one on either side of the urethral plate with no. 15 blade starting from proximal opening of the urethra till the tip of the glans penis. Skin incision is taken below the corona of glans penis to join the two incisions already taken. The skin is degloved till the base of the penis and any adhesions between the skin and the penis are cleared. Instead of the urethral plate the skin in the midline is tubularized to make the urethra. No suprapubic diversion is generally done by most surgeons. Skin cover over the penis is done by Byar's skin flaps by taking a dorsal vertical cut on the skin hood and the flaps are wrapped ventrally and sutured to each other.

The problem with the Byar's second stage urethroplasty is that the skin to make the urethral tube falls short. Hence, I made a modification in the technique and took a midline cut into the skin same as the Snodgrass cut. Then the urethral stent can accommodate easily and a wide urethra can be reconstructed from the skin. Continuous interlocking sutures of 5-0 vicryl are taken with every fifth suture a knot is taken to form the urethral tube. The suture line is covered by a Dartos flap raised from the scrotum. Total prepucial skin is rotated around the penis to cover the skin over the penile shaft.

The details of my modified technique for Tubularized incised plate urethroplasty (TIP) operation known as Warren Snodgrass repair is as follows. Operative parts prepared and draped. Glans stitch is taken with no 3-0 vicryl and held with an artery forceps. Tourniquet is applied at the base of penis with no 5 infant feeding tube. Two parallel incisions are taken one on either side of the urethral plate with no. 15 blade starting from proximal opening of the urethra till the tip of the glans penis. Skin incision is taken below the corona of glans penis to join the two incisions already taken. The skin is degloved till the base of the penis and any adhesions between the skin and the penis are cleared. The skin chordee is thus released. Chordee test is done by infiltrating saline into the corpus cavernosum through the glans penis. $90 \%$ of the times the chordee is due to the skin. If chordee is still present, then dorsal plication of the penis is done by taking two transverse incisions dorsally on tunica albuginea at the level of the chordee and suturing them together by 6-0 prolene interrupted sutures.

A snodgrass cut is taken in the midline on the uretral plate from the proximal opening of the urethra till the tip of the glans penis where the neo urethra is proposed and deepened till the corpus cavernosum of the penis. Glans wings are raised on both sides with great tissue respect. Hemostasis is achieved by cauterizing the bleeders by bipolar cautery. No 7 infant feeding tube is put into the urethral opening. Urethral construction is done by continuous interlocking sutures with 5-0 vicryl on round body needle and the mucosa of the urethral plate is inverted inside with every suture. On every 5 th suture a knot is taken and the suture line is progressed upwards till the tip of the glans penis to create neo urethra. The glans stitch suture is now fixed to the infant feeding tube.

The Midline incision is taken on the scrotum over the median raphe. The dartos fascia is dissected off both the testis on either side. The Dartos flap is elongated further by dissecting the fascia from both the testis and the skin. A tunnel is created under the skin of the penis ventrally and the Dartos flap is pulled up through the tunnel to reach the tip of the penis. Dartos flap is sutured at the tip of the penis over the neo urethra. The Dartos flap is stitched on both sides to the side of the penile body by interrupted sutures of 5-0 vicryl. Glanuloplasty is done by wrapping the glans wings on the urethra and Dartos flap and stitching with 3 interrupted sutures of 5-0 vicryl sutures. The skin hood is rotated forwards and wrapped all around the penis and sutured to the skin below the glans penis and dorsally. Thus there is no overlapping of the suture line between the urethra and the skin. The suprapubic diversion is done by open technique and no. 16 Foley's catheter is inserted into the bladder. Sofra tulle is applied over the penis all around. A gauze piece is applied over it. A compression dressing is applied over the penis with Elastoplast bandage.

\section{Follow up}

Patients were followed up as follows. First follow up was taken on one week after discharge. Urethral calibration was done to look for any obstructions in the urethra and meatus. Next follow up was taken after two weeks after discharge and again urethral calibration was done and suprapubic diversion removed. Six weeks after discharge another follow up was taken and urethral calibration was done. Majority of patients did not require any active treatment after six weeks after discharge. After six weeks patients were followed up yearly particularly for penile growth. The longest duration of follow up in my series was 10 years and the shortest was three months.

\section{Results}

The results of Tubularized incised plate urethroplasty (TIP) Warren Snodgrass repair are as follows. Fistula rate of $10 \%$ and 21\% respectively for distal and proximal hypospadias. Meatal Stenosis in $3 \%$ cases. No patient has urethral stricture and glanular dehiscence. No patient had a residual chordee. ${ }^{8}$

In other international series of 53 published studies concerning TIP cases, ${ }^{9}$ the mean meatal stenosis rate was $2.1 \%$, ranging from $0 \%$ to $17 \%$. In another review of 16 studies, ${ }^{10}$ the mean meatal stenosis rate was $3.6 \%$, ranging from $0 \%$ to $6 \%$. Complication of two staged urethroplasty are fistula rate $24 \%$, meatal stenosis $3 \%$, no patients had residual chordee and complete disruption. ${ }^{6,7,11}$ The two staged urethroplasties done with buccal mucosa as an on lay graft are very bad with $50-100 \%$ failure, ${ }^{12}$ many centers have discontinued these repairs. Hence I did not perform any of these repairs in my series.

The results of my series with 257 cases before the protocol(group A) were as follows: 33 patients $(12.8 \%)$ developed meatal stenosis for which only one urethral dilatation under GA was required. 78 patients(30.35\%) developed fistula and 18 of them closed spontaneously with conservative treatment and 60(23.34\%) required fistula repair. Total complication rate was $66.5 \%$. The results of my series with 512 cases done with Ten Commandments (group B) were as follows: 17 patients (3\%) developed meatal stenosis for which only one urethral dilatation under GA was required. Although 9 patients developed fistula $(1.75 \%) 6$ of them closed spontaneously with conservative treatment and only three required fistula repair $(0.6 \%)$. 
Total complication rate was $3.6 \%$.No patient developed a urethral stricture.

Incidance of meatal stenosis, fistula formation, fistula closure operation and total complication rate of group A was compared to group $\mathrm{B}$ and all the complications were found to be far less frequent in group B compared to group A and the difference was found to be statistically significant. Following are the details of the statistical calculations. Meatal stenosis comparative error 4.34- statistically significant. Fistula rate comparative error 5.73-statistically significant. Fistula closure rate comparative error 5.21- statistically significant. Total complication rate comparative error 5.99- statistically significant. The results of group B were compared to the series of Warren Snodgrass and Aseem Shukla who are the international authorities on the subject and have written a chapter on Hypospadias in paediatric urology text book Kelalis and King. They have a fistula rate of $10 \%$ and $21 \%$ respectively for distal and proximal hypospadias which is 17 and 35 times higher than my series. Other international series on Hypospadias have more or less similar outcomes.

\section{Discussion}

The Tubularized incised plate urethroplasty (TIP) Warren Snodgrass repair ${ }^{9,10}$ is the most common operation done for distal and mid penile hypospadias today. The simplicity of the operation made it popular worldwide. But operation has many limitations which are as follows. The midline snodgrass cut bleeds profusely post operatively leading to bleeding in and around the dressing of the penis. Blood is a culture medium and I think that it favors infection in the local area. I have seen a gush of pus coming out after removal of the urethral stent. The snodgrass cut heals with primary intention only partially and a lot by secondary intention. The cut takes about six weeks to heal completely. This raw area is infected post operatively and leads to pus discharge for many weeks. The urea in urine is irritant and every time the child passes urine, the child cries terribly and so the mother. There is a lot of edema and and pain in the local area and majority of children pass urine as "drip irrigation" and not in a stream. The Urethral meatus needs to be dilated regularly under local anesthesia or even under general anesthesia till six to twelve weeks till the snodgrass cut heals completely. I have to teach the mothers to do daily urethral dilatations with lignocaine gelly, otherwise the meatus tends to close spontaneously. A suprapubic diversion that diverts the urine stream for three weeks till the cut heals is a must to avoid the above problems of the operation. This operation has a high fistula rate and requires Dartos flap as a must to make it successful. The operation with Dartos flap and suprapubic diversion takes on an average 90 minutes to complete.

The Ten Commandments I have described are not the part of conventional Snodgrass repair and Byar's repair. These operations do not have high success rate if they are practiced as described by the authors. You need to modify them extensively for a higher success rate.

I would like to discuss the advantages of my commandments one by one as follows major commandments 1) Taking continuous interlocking sutures and a knot every 5 stitches. This kind of suturing policy is reported for the first time in Hypospadias surgery literature. These sutures are water tight and if sutures breaks in between the whole suture line does not unwind. ${ }^{8}$ There is a variety of suturing techniques reported in Hypospadias surgery. ${ }^{11}$ A technique with first layer of continuous non interlocking sutures and second layer of interrupted suture is also described with better results. Since I do a Dartos flap in every case I think that two layered technique is unnecessary. The following study compared a subcuticular uninterrupted suturing technique and full thickness interrupted sutures in eighty patients. Patients were prospectively randomized into two equal groups on the basis of the suturing technique. A neo urethra was constructed using a subcuticular uninterrupted suturing technique (group A) or using fullthickness interrupted sutures (group B). All patients were subjected to the same preoperative, operative, and follow-up protocols. Both groups were compared as regards the complication rate. The overall success rate was $90 \%$ (97.5\% for group A and $82.5 \%$ for group B). Late complications in the form of a urethrocutaneous fistula, meatal stenosis, and wound dehiscence or infection developed in eight (10\%) patients (one patient of group A and seven of group B). The subcuticular uninterrupted suturing technique seems to have a lower complication rate when compared with the use of full-thickness interrupted sutures in the repair of distal hypospadias by TIP urethroplasty. ${ }^{13}$ In my series, the continuous interlocking sutures were taken and with every fifth suture a knot was taken. It gave even better success rate of $99.4 \%$ the highest reported in medical literature so far.

The suture material used was polyglactin 910 (Vicryl). There was a controversy about suture materials to be used in hypospadias surgery. Polydioxanone (PDS) was thought to be better than polyglactin 910 (Vicryl). But it is clear from the following study that the results in hypospadias surgery are independent of the suture material. ${ }^{14}$ Three hundred and thirty-six boys undergoing a flap procedure (parameatal based, preputial tube, or onlay preputial flap) for hypospadias repair were considered for this study. The patients were stratified into two groups according to the suture material used for urethroplasty. Polyglactin (Vicryl), a polyfilament with intermediate absorption, was used in 254 group A patients, whereas polydioxanone (PDS), a monofilament with prolonged absorption, was used in 82 group B patients. The success of a one-stage repair and stricture and fistula rates were evaluated. A successful one-stage repair was achieved in $82 \%$ of the group A and in $83 \%$ of the group B patients $(p=0.97)$. No statistically significant differences were noted in fistula and/or stricture rates in the two groups, even considering each procedure separately. It concludes that that suture materials do not affect the complication rate in flap urethroplasty procedures. Appropriate technique, meticulous surgery, and surgeon experience seem to be more crucial factors.

2) Dartos Flap (Figure 1\&2) raised from scrotum. It is robust, has excellent blood supply and gives support and protection to suture line avoiding fistula formation. ${ }^{15}$ The dartos flap is fibroadipose tissue between the scrotal skin and tunica vaginalis with its vascular pedicle based at the penoscrotal Junction. The flap reaches the distal penile shaft without tension. Dartos is a vascular tissue which helps in healing. In case of fistula formation, this vascular tissue promotes healing and majority of fistulae heal without requiring a redo surgery. Dartos flap can also be raised from the prepuce but it has poor blood supply and it and the associated skin can get necrosed..$^{15,16}$ Dartos flap to protect tubularized incised plate urethroplasty is safe with a low complication rate. The neourethra is covered entirely with a layer of vascularized tissue and is good choice for preventing urethrocutaneous fistula formation. ${ }^{17}$ A large multicentric study involving 394 patients shows a drastic reduction in complications of hypospadias surgery after a Dartos flap with following results. Out of 394 patients complications occurred in 23 patients (5.83\%): fistulas $1.01 \%$ (4 cases), stenosis $0.25 \%$ ( 1 case), mild stenosis $2.53 \%$ (10 cases), dehiscence of ventral cutis $0.50 \%$ ( 2 cases) and penile torsion $1.26 \%$ ( 5 cases). All fistulae had a spontaneous resolution. ${ }^{17}$

3) Suprapubic diversion for three weeks. Healing takes about 3-4 weeks and removing urethral stent in one week time is too early. 
Urine flows in force and pressure that can easily disrupt mucosal level of healing that occur at the end of one week. Collagen is laid down at the end of a third week which gives strength to the suture line. Just adding suprapubic diversion dropped the fistula rate by about $50 \%{ }^{18,19}$ Recently there is a trend of avoiding suprapubic diversion which has no scientific rational. Suprapubic diversion shall be necessary till we have a breakthrough in wound healing where three weeks healing can be preponed in one week. I had many patients who did not have fistula initially but developed after one week or so. It proves that although initially there is no fistula, due to the force and pressure of urine it develops afterwards by disrupting the mucosal level of healing without collagen to support it. The following study on 105 patients had complications such as fistula and meatal stenosis significantly less in a group in which suprapubic diversion was used. In 56 of the 105 patients in whom suprapubic diversion was used, four $(7.14 \%)$ had fistulae and three $(5.35 \%)$ had meatal stenosis, in contrast to a fistula rate of $14.28 \%$ and meatal stenosis rate of $12.24 \%$ in patients that urethral stent is used for urinary drainage. It proves that the use of suprapubic diversion is advantageous for the outcome of one stage hypospadias repair in relation to fistula occurrence and meatal stenosis. ${ }^{18}$

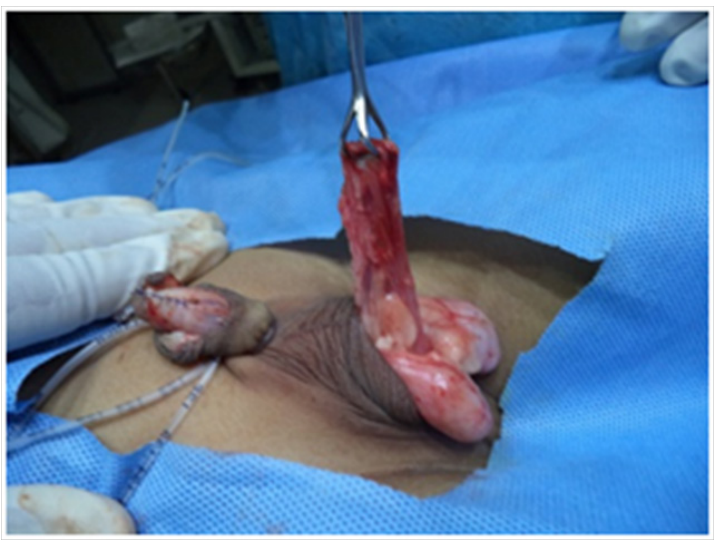

Figure I Dartos Flap raised from the scrotum.

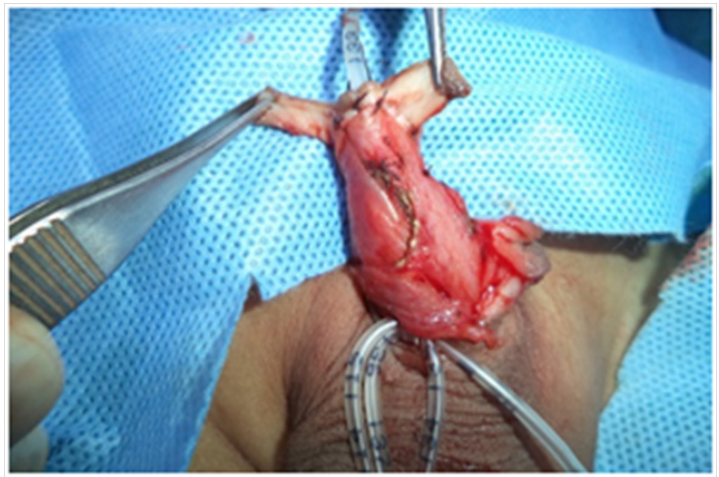

Figure 2 Dartos Flap applied to the penile shaft.

In another randomized clinical trial out of 192 patients, fistula was reported in eight patients (12.7\%) of urethral catheter group A, while it was observed in three patients $(2.3 \%)$ of suprapubic diversion groups B. Meatal stenosis was reported in eight patients in group B (12.7\%; nonstented group) versus three patients of both groups A (2.4\%; stented groups) with a statistically significant difference $(\mathrm{p}<0.05) .{ }^{19}$ It proves that Suprapubic diversion is an important step in hypospadias repair as it provides a better success rate with a significantly lower rate of occurrence of fistula. 3) Rotating total prepucial skin instead of Byar's skin flaps.It is Popularized by Dr. Asopa and Dr. Duckett in 1970 (Figure 3). dorsally cutting the prepucial hood can jeopardize the blood supply of either of skin flaps and it can get necrosed leading to failure of the operation. ${ }^{20,21}$ Another problem is that suture line of urethra crosses with the skin which is a violation of basic principles of surgery. It leads to a higher chance of fistula formation. ${ }^{20}$ In the following study, Byars' method was used in 8 of these to cover the defect and the Ombredanne method in the remaining 2. Necrosis developed in the skin flap of 5 cases $(50 \%)$ (4 Byars and 1 Ombredanne). Urethral fistula developed in the other patient whose necrosis was deeper. The mean hospital stay was 7 days for patients without necrosis and 14 for those with necrosis (between 13 and 15 days)..$^{20,21}$

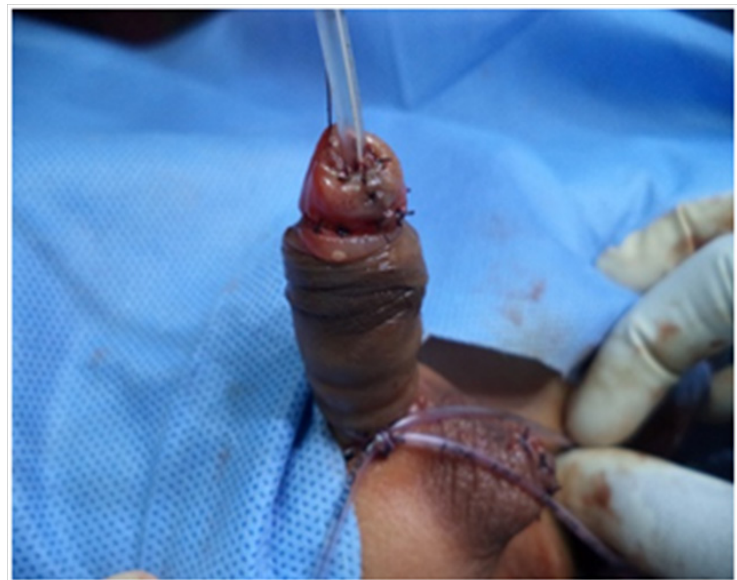

Figure 3 Photo showing completed repair.

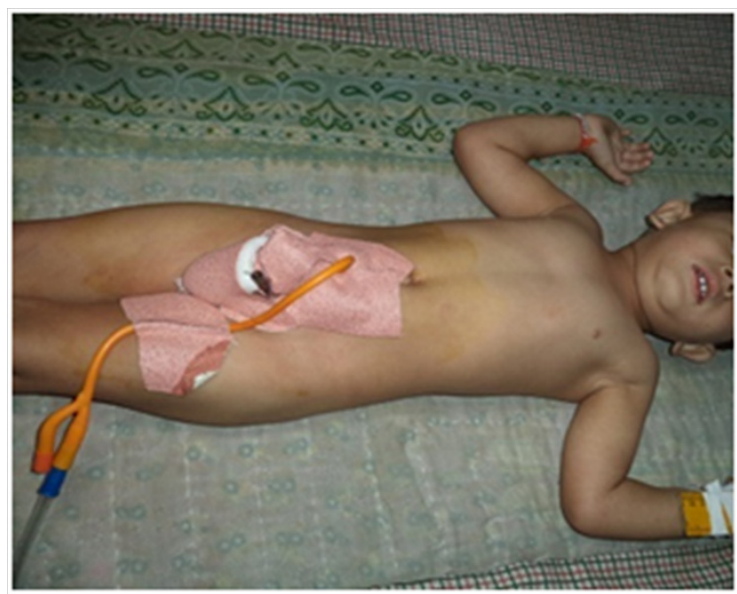

Figure 4 The dressing policy.

Devascularization of the flap or graft is a major complication and reported incidence is $7 \%$. Various causes of devascularization are damage to vascular supply while raising the flap, hematoma, infection, vascular spasm, and tight pressure dressing The necrosis may be superficial and dermal (commonly seen due to pressure dressing) and heal without permanent damage.The viability of the neourethra can be evaluated by simply looking at the outer face of the flap in case of double island flap urethroplasties. It can be prevented by proper graft design, good surgical technique maintaining the proper plane of dissection, good hemostasis to avoid hematoma, administration of broad spectrum antibiotics to prevent infection, avoiding tight dressings, local application of nitroglycerin ointment to prevent vasospasm and counter incisions. If any part of graft or 
flap is devitalized, it should be judiciously debrided. If area of devascularization is small and pedicle of the flap is intact, satisfactory result can still be obtained without reoperating. But major dehiscence, e.g. glanular wing dehiscence in a hypospadias repair leading to glanular breakdown, would need further operative intervention. ${ }^{21}$

5) Tourniquet instead of adrenaline infiltration. Adrenaline can give false sense of no bleeding and post operatively big hematomas form that will disrupt the repair. If adrenaline accidently enters blood stream, it can lead to serious cardiac arrhythmias. Adrenaline can also lead to necrosis of the skin around areas of infiltration..$^{22}$. Adrenaline if enters the corpus cavernosum can rarely lead to penile gangrene which is a grievous injury in the medico legal law. Although a tourniquet is frequently used in penile surgery there is still no consensus on safe application time. The following study was done to investigate the effect of malondialdehyde (MDA) levels and histological changes in skin flaps after penile tourniquet application and epinephrine injection. A total of 36 male white New Zealand rabbits were randomly divided into six groups each containing six animals. A Mathieu-like flap was raised in all of the groups and a tourniquet was applied and the penis was subjected to ischemia for 10, 20 and $40 \mathrm{~min}$ in groups 1,2 and 3, respectively. The flaps were then allowed to re perfuse for $5 \mathrm{~min}$. Biopsies for MDA measurement were harvested in these groups. Subcutaneous 1/200,000 epinephrine was injected into penile skin in group 4 and 5 rabbits and biopsies for MDA measurement were harvested 10 and $40 \mathrm{~min}$ after injection. The control group was anesthetized without tourniquet usage or epinephrine injection. Specimens taken from the harvested flaps of all groups were submitted for histological evaluation. The mean MDA levels in all experimental groups were higher than in the control group and the difference was statistically significant. Edema, congestion and extravasation were observed in groups 1, 2 and 3. Minimal congestion and edema were observed in group 4 and severe edema and extravasation in group 5. Tourniquet usage for a duration of less than $10 \mathrm{~min}$ is clearly safer than prolonged usage. Epinephrine injection to penile skin may show a deleterious effect on wound healing. ${ }^{22}$

1. The minor commandments dressing policy (Figure 4) the penis is covered with a sofra tulle and a gauze piece is applied over it. Compression dressing is given by Elastoplasts. It takes care of postoperative bleeding and hematoma formation. Such a dressing also protects the repair from any possible injuries in the post-operative period. Dressings must be changed every 48 hours as it brings to your notice a lot of problems much earlier. A subcutaneous hematoma, wound infection, active bleeding, skin flap necrosis is noticed earlier and can be handled immediately. Although a variety of dressing policies are reported in Hypospadias surgery covering the penis all around with Elastoplast as shown in figure 4 was most protective against any injuries that can occur in postoperative period. ${ }^{23}$ I think Hypospadias dressing to be the most delicate and skillful in entire pediatric surgery, hence all dressings were done by me personally instead of leaving it to the assistants. Many authors do not give importance to these protocols which may be the real reason of failure of surgery. The dressings can be kept even after removal of the urethral stent and patients were discharged with it. It protects the repair in the post-operative period. The children can pass urine through the dressings comfortably at home.

The following study has a similar dressing policy as my series. The sterile tubular net bandage has been applied immediately after hypospadias repairing operations in 126 cases from age 1 to age 3 . After urethroplasty, a catheter was inserted in the new urethra, and then the tubular net bandage was applied on the shaft of the penis. The dressing was changed on the third and seventh postoperative days and replaced by a new set. If necessary, the dressing could be changed while inspection. After catheter was removed on the fifth postoperative day, the patient could void with the dressing on the penis. The tubular elastic net bandage is gently compressive, comfortable, and not inclined to slip off. It is well tolerated by children and does not affect the normal voiding after the catheter removed. The dislodgement of the bandage has occurred in eight cases. The dislodgement might be due to the restless movement of the legs after operation. The elastic dressing did not disturb the normal voiding function. The children could void with the dressing on the penis. This helped children to tolerate the penile dressing and reduced their psychological stress. ${ }^{24}$

2. High protein diet. Despite good Hypospadias repair I got some failures due to poor wound healing. I started high protein diet to the child before, during and after surgery in the form of eggs or protinex powder that made dramatic difference to healing and results. ${ }^{25}$ Following article discusses link between diet and wound healing. Nutrition is a crucial aspect of a holistic approach to the healing of wounds. Poor nutritional status and support can delay the wound healing process or cause inadequate healing when nutritional deficiencies are not corrected. Nutritional screening is an important part of the overall clinical assessment of patients. Patients' nutritional requirements may need to be met through the use of nutritionally complete supplements or, if oral intake is poor, with enteral or parenteral nutrition. ${ }^{26}$

3. Tab Oxybutynin given in twice a day schedule. Violent bladder contractions can lead to peri urethral gush of urine and a possible fistula formation. The child complains of severe pain post operatively due to bladder contractions. It leads to major morbidity to the patient after hypospadias surgery. Oxybutynin has been used for the management of detrusor overactivity for over 30 years and has withstood medical scrutiny and the test of time throughout the world. Although several agents in the class of bladder relaxants have only recently been studied, oxybutynin's effectiveness in reducing urinary frequency and urge urinary incontinence is unquestioned in the medical literature. Oxybutynin is extremely safe and effective in almost every population including children. With more preparations available and more dosing flexibility than any other anticholinergic medication on the market, oxybutynin remains the "gold standard" for first line therapy for patients who have detrusor overactivity. ${ }^{27}$

4. Using bipolar cautery to cauterize all major bleeders. Many surgeons do not use electro cautery in Hypospadias surgery as serious injuries are reported to penis after using mono polar cautery. But if all major bleeders are not cauterized, post operatively a major hematoma can form that can destroy the repair. Hence only bipolar cautery should be used in Hypospadias surgery. ${ }^{28}$ In the following study of 60 patients in Group A included 30 patients in whom the tourniquet, rolled rubber glove, was applied around the base of the penis. The maximum application time was $40 \mathrm{~min}$; if the operation lasted longer than 40 min the tourniquet was released for $10 \mathrm{~min}$. Group B included 30 patients in whom the operation was performed without using a tourniquet and hemostasis was obtained by preoperative infiltration of the incision site with adrenaline (1/20 000) and bipolar cautery throughout the procedure. Early postoperative hematomas occurred in $13.3 \%$ of group A and $6.6 \%$ of group B patients. Diffuse penile edema appeared in $23.3 \%$ of group A and $10 \%$ of group B patients. Postoperative bleeding occurred in $6.6 \%$ of group $\mathrm{A}$ and $3.3 \%$ of group B patients. One patient in group A developed wound infection. However, there was no significant statistical difference between the two groups with regard to early postoperative complications. Meticulous hemostasis is an important requisite for any form of surgery. It reduces blood loss and ensures good operating conditions. 
During hypospadias repair, the best option for maintenance of effective hemostasis without permanent tissue injury has not been determined. However, it is generally accepted that the use of hemostasis methods should be kept to a minimum to avoid ischemic injuries.

In the following study two different methods for obtaining hemostasis during hypospadias surgery ,vasoconstrictors and bipolar diathermy (group A) versus tourniquet only (group B) were compared. The average duration of surgery in group A was 76.66 versus $88.50 \mathrm{~min}$ in group B. The duration of surgery was significantly different in the two groups $(\mathrm{P}<0.05)$. The shorter times in group A may be attributed to the clearer operative field consequent to tourniquet application. The incidence of postoperative diffuse penile edema, hematoma, bleeding, and stenosis was higher in group A when compared with group B. These results are in accordance with other studies. ${ }^{29}$

5. Ornidazole cover. Despite higher antibiotic coverage I was getting infections which were destroying the repair. Adding anaerobic coverage..$^{11}$ substantially dropped the infection rate. ${ }^{30}$ The other antibiotics used along with Ornidazole were Ceftriaxone and Amikacin. Anaerobes have been involved in many different types of urinary infections. The anaerobes recovered in genital areas are Gram negative bacilli (including Bacteroides fragilis and pigmented Prevotella and Porphyromonas sp.), Clostridium sp., anaerobic Grampositive cocci and Actinomyces $s p$. In many cases, they are found mixed with coliforms or streptococci. The presence of anaerobes in and around genital area requires the administration of antimicrobial therapy that is effective against these organisms. These antimicrobials include metronidazole, chloramphenicol, clindamycin, a carbapenem, cefoxitin and the combination of penicillin and a beta-lactamase inhibitor. ${ }^{29,30}$

\section{Conclusion}

This multidisciplinary approach improved the success rate of hypospadias surgery dramatically to $99.4 \%$. The complication rate in group B was far less than that of group A and it was found to be statistically very significant. It is the world's highest reported success rate in Hypospadias surgery. Since the Author Warren Snodgrass and others did not follow any of the above Commandments in their series, there is a reason to follow them for a much better outcome. Since the results of Hypospadias surgery are comparatively poor in general, we must keep an open mind and look forward for better alternatives. In the bibliography section I have collected references that clearly support each of my commandments. Many pediatric surgeons are deciding protocols based on dictations given by the patient's relatives and their own ideas rather than science to save money and operative time. It is dangerous to the patient community. I believe that similar results are achievable by any pediatric surgeon by following the Ten Commandments.

\section{Acknowledgments}

None.

\section{Conflicts of interest}

The authors declare no conflicts of interest.

\section{References}

1. Snodgrass W. Tubularized incised plate uretheroplasty for distal Hypospadias. J Urol. 1994;151(2):464-465.

2. Snodgrass W, Koyle M, Manzoni G, et al. Ehrlich Tubularized incised plate repair for proximal hypospadias. J Urol. 1998;159(6):2129-2131.
3. Gershbaum MD, Stock JA, Hanna MK. A case for 2-stage repair of perineoscrotal hypospadias with severe chordee. J Urol. 2002;68(4 pt 2):1727-1728

4. Bracka A. A versatile two-stage hypospadias repair. Br J Plast Surg. 1995;48(6):345-352.

5. Johal NS, Nitkunan T, O'Malley K, et al. The two-stage repair for severe primary hypospadias. Eur Urol. 2006;50(2):366-371.

6. Telfer JR, Quaba AA, Kwai Ben I, et al. An investigation into the role of waterproofing in a two-stage hypospadias repair. Br J Plast Surg. 1998;51(7):542-546.

7. Ramanathan C. Three-year experience of hypospadias surgery:Bracka's method. Indian J Plast Surg. 2006;39(2):130-135.

8. Ulman I, Erikçi V, Avanoğlu A, et al. The effect of suturing technique and material on complication rate following hypospadias repair. Eur $J$ Pediatr Surg. 1997;7(3):156-157.

9. Snodgrass W, Koyle M, Manzoni G, et al. Tubularized incised plate hypospadias repair: Results of a multicenter experience. $J$ Urol. 1996;156(2 pt 2):839-841.

10. Elbakry A. Further experience with the tubularized-incised urethral plate technique for hypospadias repair. BJU Int. 2002;89(3):291-294.

11. M Abdelmoneim Gafa. Two different suturing techniques in distal hypospadias repair using tubularized incised plate urethroplasty:a prospective randomized study. Annals of Pediatric Surgery. 2013;9(3):117-121.

12. Hensle TW, Kearney MC, Bingham JB. Buccal mucosa grafts for hypospadias surgery: Long-term results. J Urol. 2002;168(4 pt 2):1734 1736.

13. AbouZeid AA. Modified Byars' flaps for securing skin closure in proximal and mid-penile hypospadias. Ther Adv Urol. 2011;3(6):251256

14. Cimador M, Castagnetti M, Milazzo M, et al. Suture materials: do they affect fistula and stricture rates in flap urethroplasties? Urol Int. 2004;73(4):320-324.

15. Churchill BM, Van Savage JG, Khoury AE, et al. The dartos flap as an adjunct in preventing urethrocutaneous fistulas in repeat hypospadias surgery. J Urol. 1996;156(6):2047-2049.

16. Soygur T, Arikan N, Zumrutbas AE, et al. Snodgrass hypospadias repair with ventral based dartos flap in combination with mucosal collars. Eur Urol. 2009;47(6):879-888.

17. Bertozzi M, Yildiz A, Kamal B, et al. Multicentric experience on double dartos flap protection in tubularized incised plate urethroplasty for distal and midpenile hypospadias. Pediatr Surg Int. 2011;27(12):1331-1336.

18. Demirbilek S, Atayurt HF. One-stage hypospadias repair with stent or suprapubic diversion: which is better? JPediatr Surg. 1997;32(12):17111712.

19. Chen CC. Urinary diversion in hypospadias repair. J Formos Med Assoc. 1993;92(12):1057-1059.

20. Bakal Ü, Abeș M, Sarac M (2015) Necrosis of the ventral penile skin flap:a complication of hypospadias surgery in children. Adv Urol 2015:452870.

21. Bhat A, Mandal AK. Acute postoperative complications of hypospadias repair. Indian J Urol. 2008;24(2):241-248.

22. Cakmak M, Caglayan F, Kisa U, et al. Tourniquet application and epinephrine injection to penile skin:is it safe? Urol Res. 2002;30(4):268272.

23. Tan KK, Reid CD. A simple penile dressing following hypospadias surgery. Br J Plast Surg. 1990;43(5):628-629. 
24. Todorovic V. Food and wounds:nutritional factors in wound formation and healing. Br J Community Nurs. 2002;43(4):46-48.

25. Demling RH. Nutrition, Anabolism, and the Wound Healing Process:An Overview. Eplasty. 2009;9:e9.

26. Rasha Kassem, Khalid Shreef, Hazem Eltayeb, et al. The use of tourniquet versus bipolar cautery as hemostatic aid in distal hypospadias repair in children: a multicentric study. The Egyptian Journal of Surgery. 2017;36(1):58-61.

27. Diokno A, Ingber M. Oxybutynin in detrusor overactivity. Urol Clin North Am. 2006;33(4):439-445.
28. Uzun G, Ozdemir Y, Eroglu M, et al. Electrocautery-induced gangrene of the glans penis in a child following circumcision. BMJ Case Rep. 2012 .

29. Brook I. Urinary tract and genito-urinary suppurative infections due to anaerobic bacteria. Int J Urol. 2004;11(3):133-141.

30. Kanaroglou N. Antibiotic prophylaxis in hypospadias repair:It's time to re-evaluate. Can Urol Assoc J. 2014;8(7-8):241. 